\title{
HANKEL OPERATORS ON THE BERGMAN SPACE OF THE UNIT POLYDISC
}

\author{
HUIPING LI
}

(Communicated by Theodore W. Gamelin)

\begin{abstract}
Let $D$ be the unit polydisc in $\mathbb{C}^{n}$. Let $H^{2}(D)$ be the Bergman space of $D$. In this paper, by using the integral representations of solutions to the $\bar{\partial}$-equations, we give function theoretic characterizations of functions $f \in L^{2}(D)$ such that the Hankel operators $H_{f}$ from the Bergman space to $L^{2}(D)$ are bounded and compact, respectively.
\end{abstract}

\section{INTRODUCTION}

Let $D$ be the unit polydisc in $\mathbb{C}^{n}$. Let $H^{2}(D)$ be the Bergman space consisting of square integrable holomorphic functions in $D$ and $P$ the Bergman projection from $L^{2}(D)$ to $H^{2}(D)$ defined by $P(f)(z)=\int_{D} f(w) K(z, w) d v(w)$, where $K(z, w)$ is the Bergman kernel of $D$ and $d v$ the usual Lebesgue measure on $\mathbb{C}^{n}$. For $f \in L^{2}(D)$, the Hankel operator $H_{f}$ from $H^{2}(D)$ to $L^{2}(D)$ is defined by $H_{f}=(I-P) M_{f}$, where $M_{f}$ is the multiplication operator. For the unit disc $\Delta$ in the complex plane $\mathbb{C}$, Stroethoff [11] characterized bounded functions $f$ such that $H_{f}$ are compact. Luecking [7] characterized functions $f \in L^{2}(\Delta)$ such that $H_{f}$ are bounded and compact respectively. At the end of [7], Luecking pointed out the difficulties in extending his results to domains in $\mathbb{C}^{n}$ and to the weighted Bergman spaces. By using the integral representations of solutions of the $\bar{\partial}$-equations, we overcome those difficulties. In [6] we characterized functions $f \in L^{2}(\Omega)$ such that $H_{f}$ are, respectively, bounded and compact on bounded strongly pseudoconvex domains $\Omega \subset \mathbb{C}^{n}$. In this paper, we are going to solve those problems on polydiscs in $\mathbb{C}^{n}$.

For $z, w \in D$, let $\beta(z, w)$ be the Bergman distance between them. For $z \in D$ and $r>0$, the Bergman metric ball $B(z, r)=\{w \in D: \beta(z, w)<r\}$. Denote by $|B(z, r)|$ the Lebesgue measure of $B(z, r)$. For $f \in L^{2}(D)$, write

$$
F_{r}(z)^{2}=\inf \left\{|B(z, r)|^{-1} \cdot \int_{B(z, r)}|f(w)-h(w)|^{2} d v(w): h \in H^{2}(D)\right\} .
$$

Received by the editors May 20, 1992 and, in revised form, July 9, 1992.

1991 Mathematics Subject Classification. Primary 47B35; Secondary 32F15.

Research partially supported by a grant of the National Science Foundation. 
For a $(p, q)$-form $H(z)=\sum H_{I, J}(z) d z_{I} \wedge d \bar{z}_{J}$ with locally integrable coefficients on $D$, write $|H(z)|=\sum\left|H_{I, J}\right|$. As usual,

$$
\bar{\partial} H(z)=\sum_{k=1}^{n} \sum \partial H_{I, J} / \partial \bar{z}_{k} d \bar{z}_{k} \wedge d z_{I} \wedge d \bar{z}_{J},
$$

where, for $1 \leq k \leq n, \partial / \partial \bar{z}_{k}=(1 / 2) \cdot\left(\partial / \partial x_{k}+i \cdot \partial / \partial y_{k}\right)$. If $H_{I, J}$ are not differentiable, the derivatives should be understood in the distribution sense.

Now we state our main theorems.

Theorem A. Let $f \in L^{2}(D)$. Then the following are equivalent.

(1) $H_{f}$ is bounded.

(2) For each $r>0, F_{r}$ is bounded on $D$.

(3) For some $r>0, F_{r}$ is bounded on $D$.

(4) $f$ admits a decomposition $f=f_{1}+f_{2}$ with $f_{2} \in C^{1}(D) \cap L^{2}$ and $f_{1} \in L^{2}(D)$ such that, for each $1 \leq i \leq n,\left(\partial f_{2} / \partial \bar{z}_{i}\right) \cdot\left(1-\left|z_{i}\right|^{2}\right) \in L^{\infty}(D)$ and, for some $r>0$,

$$
G_{r}(z)=|B(z, r)|^{-1} \cdot \int_{B(z, r)}\left|f_{1}\right|^{2} d v \in L^{\infty}(D) .
$$

Theorem B. Let $f \in L^{2}(D)$. Then the following are equivalent.

(1) $H_{f}$ is compact.

(2) For each $r>0, F_{r}(z) \rightarrow 0$ as $z \rightarrow \partial D$.

(3) For some $r>0, F_{r}(z) \rightarrow 0$ as $z \rightarrow \partial D$.

(4) $f$ admits a decomposition $f=f_{1}+f_{2}$ with $f_{2} \in C^{1}(D) \cap L^{2}$ and $f_{1} \in L^{2}(D)$ such that, for each $1 \leq i \leq n,\left(\partial f_{2} / \partial \bar{z}_{i}\right) \cdot\left(1-\left|z_{i}\right|^{2}\right) \rightarrow 0$ as $z \rightarrow \partial D$ and, for some $r>0$,

$$
G_{r}(z)=|B(z, r)|^{-1} \cdot \int_{B(z, r)}\left|f_{1}\right|^{2} d v \rightarrow 0 \quad \text { as } z \rightarrow \partial D .
$$

In $\S 2$ we will discuss the Hankel operators $H_{f}$ with $f \in C^{1}(D)$ by using the integral representations of solutions to the $\bar{\partial}$-equations. In $\S 3$ we will prove the main theorems and the $L^{p}$-versions of Theorems A and B.

To make the ideas clear, we shall only prove the theorems for the unit polydisc in $\mathbb{C}^{2}$. For general cases, one can obtain the results by proceeding in the same way.

Throughout this paper, we will use the letter $C$ to denote constants, and they may change from line to line.

\section{HANKEL OPERATORS WITH $C^{1}$ SYMBols}

From now on, we shall assume that $D$ is the unit polydisc in $\mathbb{C}^{2}$. To simplify notation, for $k, m \geq 2$, write

$$
\begin{aligned}
& L_{1, k, m}(z, w)=(2 \pi i)^{-1} \cdot\left(1-\left|w_{1}\right|^{2}\right)^{k} \cdot\left(1-z_{1} \bar{w}_{1}\right)^{-k} \cdot\left(z_{1}-w_{1}\right)^{-1} d \bar{w}_{1} \wedge d w_{1} \\
& L_{2, k, m}(z, w)=(2 \pi i)^{-1} \cdot\left(1-\left|w_{2}\right|^{2}\right)^{m} \cdot\left(1-z_{2} \bar{w}_{2}\right)^{-m} \cdot\left(z_{2}-w_{2}\right)^{-1} d \bar{w}_{2} \wedge d w_{2}
\end{aligned}
$$




$$
\begin{aligned}
& L_{3, k, m}=(4 \pi)^{-2} \cdot\left(\frac{1-\left|w_{1}\right|^{2}}{1-z_{1} \bar{w}_{1}}\right)^{k} \\
& \cdot\left(\frac{1-\left|w_{2}\right|^{2}}{1-z_{2} \bar{w}_{2}}\right)^{m} \frac{\left(\bar{w}_{1}-\bar{z}_{1}\right) d \bar{w}_{2}-\left(\bar{w}_{2}-\bar{z}_{2}\right) d \bar{w}_{1}}{|z-w|^{4}} \wedge d w_{1} \wedge d w_{2} ; \\
& L_{4, k, m}= 1 /\left(4 \pi^{2}\right)\left\{m \cdot \frac{\left(1-\left|w_{1}\right|^{2}\right)^{k}}{\left(1-z_{1} \bar{w}_{1}\right)^{k} \cdot\left(z_{1}-w_{1}\right)}\right. \\
&\left.\cdot \frac{\left(1-\left|w_{2}\right|^{2}\right)^{m-1}}{\left(1-z_{2} \bar{w}_{2}\right)^{m+1}} \cdot \frac{\left|w_{2}-z_{2}\right|^{2}}{|w-z|^{2}}\right\} d \bar{w}_{2} \wedge d w_{1} \wedge d w_{2} ; \\
& L_{5, k, m}=1 /\left(4 \pi^{2}\right)\{k \cdot \frac{\left(1-\left|w_{2}\right|^{2}\right)^{m}}{\left(1-z_{2} \bar{w}_{2}\right)^{m} \cdot\left(z_{2}-w_{2}\right)} \\
&\left.\cdot \frac{\left(1-\left|w_{1}\right|^{2}\right)^{k-1}}{\left(1-z_{1} \bar{w}_{1}\right)^{k+1}} \cdot \frac{\left|w_{1}-z_{1}\right|^{2}}{|w-z|^{2}}\right\} d \bar{w}_{1} \wedge d w_{1} \wedge d w_{2} .
\end{aligned}
$$

Let $\Delta$ be the unit disc in the complex plane $\mathbb{C}$.

Lemma 2.1. For $z, w \in \Delta$, if $t>0$ and $0<\varepsilon<1$, then

$$
\begin{gathered}
\int_{\Delta}|1-z \cdot \bar{w}|^{-1} \cdot|z-w|^{-1} \cdot\left(1-|w|^{2}\right)^{-\varepsilon} d v(w) \leq C \cdot\left(1-|z|^{2}\right)^{-\varepsilon} \\
\int_{\Delta}|1-z \cdot \bar{w}|^{-2} \cdot\left(1-|w|^{2}\right)^{-\varepsilon} d v(w) \leq C \cdot\left(1-|z|^{2}\right)^{-\varepsilon}
\end{gathered}
$$

and

$$
\int_{\Delta}|1-z \cdot \bar{w}|^{-2-t} d v(w) \leq C \cdot\left(1-|z|^{2}\right)^{-t} .
$$

Proof. Let $u=(w-z) /(1-w \cdot \bar{z})$. Then

$$
\begin{aligned}
\int_{\Delta} \mid 1 & -\left.z \cdot \bar{w}\right|^{-1} \cdot|z-w|^{-1} \cdot\left(1-|w|^{2}\right)^{-\varepsilon} d v(w) \\
& \leq C \cdot\left(1-|z|^{2}\right)^{-\varepsilon} \cdot \int_{\Delta}\left|u \cdot(1+u \cdot \bar{z})^{2-2 \varepsilon} \cdot\left(1-|u|^{2}\right)^{\varepsilon}\right|^{-1} d v(u) \\
& \leq C \cdot\left(1-|z|^{2}\right)^{-\varepsilon} \cdot\left(1+\int_{\Delta}|1+u \cdot \bar{z}|^{-2+2 \varepsilon} \cdot\left(1-|u|^{2}\right)^{-\varepsilon} d v(u)\right) .
\end{aligned}
$$

By using the method in the proof of [1, Lemma 4], it follows that the integral in the last inequality of (2.4) is finite. This finishes the proof of (2.1).

The proofs of (2.2) and (2.3) are similar. We omit the details here. Q.E.D.

Lemma 2.2. If $\gamma(z)=\gamma_{1}(z) d \bar{z}_{1}+\gamma_{2}(z) d \bar{z}_{2}$ is a $\bar{\partial}$-closed $(0,1)$ form with $\left|\gamma_{1}(z)\right| \cdot\left(1-\left|z_{1}\right|^{2}\right)+\left|\gamma_{2}(z)\right| \cdot\left(1-\left|z_{2}\right|^{2}\right) \in L^{1}(D)$, then for each pair $k, m \geq 2$

$$
\begin{aligned}
u(z)= & T(\gamma)(z) \\
= & \int_{\Delta} \gamma_{1}\left(w_{1}, z_{2}\right) \cdot L_{1, k, m}(z, w)+\int_{\Delta} \gamma_{2}\left(z_{1}, w_{2}\right) \cdot L_{2, k, m}(z, w) \\
& +\int_{D} \gamma(w) \wedge L_{3, k, m}(z, w)+\int_{D} \gamma(w) \wedge\left\{L_{4, k, m}(z, w)-L_{5, k, m}(z, w)\right\}
\end{aligned}
$$

is a solution to the equation $\bar{\partial} u=\gamma$. 
Proof. If the coefficients of $\gamma$ are in $C^{1}(\bar{D})$, the result was proved in [3]. By using the Fubini Theorem and the estimates in Lemma 2.1, one can easily prove that

$$
\|T(\gamma)\|_{1} \leq C \cdot\left(\left\|\gamma_{1} \cdot\left(1-\left|z_{1}\right|^{2}\right)\right\|_{1}+\left\|\gamma_{2} \cdot\left(1-\left|z_{2}\right|^{2}\right)\right\|_{1}\right) .
$$

By a standard regularization argument [10], the result follows.

For $f \in C^{1}(D)$, write $\Psi_{1}(z)=\partial f(z) / \partial \bar{z}_{1} \cdot\left(1-\left|z_{1}\right|^{2}\right), \Psi_{2}(z)=\partial f(z) / \partial \bar{z}_{2}$. $\left(1-\left|z_{2}\right|^{2}\right)$.

Lemma 2.3. For $f \in C^{1}(D) \cap L^{2}(D)$, if $\partial f(z) / \partial \bar{z}_{1} \cdot\left(1-\left|z_{1}\right|^{2}\right)$ and $\partial f(z) / \partial \bar{z}_{2}$. $\left(1-\left|z_{2}\right|^{2}\right)$ are bounded in $D$, then $T_{f}(g)=T(\bar{\partial}(f \cdot g))=T(g \bar{\partial} f)$ is bounded from $H^{2}(D)$ to $L^{2}(D)$, where $T$ is the operator given in Lemma 2.2.

Proof. For $g \in H^{\infty}(D)$, the space of bounded holomorphic funcitons in $D$, we have

$$
\begin{aligned}
& g\left(w_{1}, z_{2}\right)=(2 \pi i)^{-1} \int_{\Delta} g\left(w_{1}, w_{2}\right) /\left(1-z_{2} \bar{w}_{2}\right)^{2} d \bar{w}_{2} \wedge d w_{2}, \\
& g\left(z_{1}, w_{2}\right)=(2 \pi i)^{-1} \int_{\Delta} g\left(w_{1}, w_{2}\right) /\left(1-z_{1} \bar{w}_{1}\right)^{2} d \bar{w}_{1} \wedge d w_{1} .
\end{aligned}
$$

Thus,

$$
\begin{aligned}
T_{f}(g)= & T(g \cdot \bar{\partial} f) \\
= & (2 \pi i)^{-1} \int_{D} g(w) \cdot \frac{\partial f\left(w_{1}, z_{2}\right)}{\partial \bar{w}_{1}} \cdot \frac{L_{1, k, m}(z, w)}{\left(1-z_{2} \bar{w}_{2}\right)^{2}} \wedge d \bar{w}_{2} \wedge d w_{2} \\
& +(2 \pi i)^{-1} \int_{D} g(w) \cdot \frac{\partial f\left(z_{1}, w_{2}\right)}{\partial \bar{w}_{2}} \cdot \frac{L_{2, k, m}(z, w)}{\left(1-z_{1} \bar{w}_{1}\right)^{2}} d \bar{w}_{1} \wedge d w_{1} \\
& +\int_{D} g \cdot \bar{\partial} f(w) \wedge L_{3, k, m}(z, w) \\
& +\int_{D} g \cdot \bar{\partial} f(w) \wedge\left\{L_{4, k, m}(z, w)-L_{5, k, m}(z, w)\right\}
\end{aligned}
$$

Note that $\left(1-\left|w_{j}\right|^{2}\right) \leq \frac{1}{2} \cdot\left|1-z_{j} \bar{w}_{j}\right|$ for $z_{j}, w_{j} \in \Delta$. One can easily check that

$$
\begin{aligned}
& \left|\left(\partial f\left(w_{1}, z_{2}\right) / \partial \bar{w}_{1}\right) \cdot L_{1, k, m}(z, w) /\left[\left(1-z_{2} \bar{w}_{2}\right)\right]^{2}\right| \\
& \quad \leq C \cdot\left\|\Psi_{1}\right\|_{\infty} /\left|\left(1-z_{1} \bar{w}_{1}\right)\left(z_{1}-w_{1}\right)\left(1-z_{2} \bar{w}_{2}\right)^{2}\right| \\
& \left|\left(\partial f\left(z_{1}, w_{2}\right) / \partial \bar{w}_{2}\right) \cdot L_{2, k, m}(z, w) /\left[\left(1-z_{1} \bar{w}_{1}\right)\right]^{2}\right| \\
& \quad \leq C \cdot\left\|\Psi_{2}\right\|_{\infty} /\left|\left(1-z_{2} \bar{w}_{2}\right)\left(z_{2}-w_{2}\right)\left(1-z_{1} \bar{w}_{1}\right)^{2}\right| \\
& \left|\bar{\partial} f \wedge L_{3, k, m}\right| \\
& \quad \leq C \cdot\left(\left\|\Psi_{1}\right\|_{\infty}+\left\|\Psi_{2}\right\|_{\infty}\right)\left(\left|1-z_{1} \bar{w}_{1}\right|^{-1}+\left|1-z_{2} \bar{w}_{2}\right|^{-1}\right) \cdot|z-w|^{-3}, \\
& \left|\bar{\partial} f \wedge L_{4, k, m}\right| \leq C \cdot\left\|\Psi_{1}\right\|_{\infty} /\left|\left(1-z_{1} \bar{w}_{1}\right)\left(z_{1}-w_{1}\right)\left(1-z_{2} \bar{w}_{2}\right)^{2}\right|, \\
& \left|\bar{\partial} f \wedge L_{5, k, m}\right| \leq C \cdot\left\|\Psi_{2}\right\|_{\infty} /\left|\left(1-z_{2} \bar{w}_{2}\right)\left(z_{2}-w_{2}\right)\left(1-z_{1} \bar{w}_{1}\right)^{2}\right| .
\end{aligned}
$$


By Lemma 2.1, it follows that for $1 \leq i, j \leq 2$ and $i \neq j$

$$
\begin{aligned}
& \int_{D}\left|\left(1-z_{i} \bar{w}_{i}\right)\left(z_{i}-w_{i}\right)\left(1-z_{j} \bar{w}_{j}\right)^{2}\left(1-\left|w_{i}\right|^{2}\right)^{\varepsilon}\left(1-\left|w_{j}\right|^{2}\right)^{\varepsilon}\right|^{-1} d v(w) \\
& \quad \leq C\left(1-\left|z_{i}\right|^{2}\right)^{-\varepsilon}\left(1-\left|z_{j}\right|^{2}\right)^{-\varepsilon}, \\
& \int_{D}\left|\left(1-z_{i} \bar{w}_{i}\right)\left(z_{i}-w_{i}\right)\left(1-z_{j} \bar{w}_{j}\right)^{2}\left(1-\left|z_{i}\right|^{2}\right)^{\varepsilon}\left(1-\left|z_{j}\right|^{2}\right)^{\varepsilon}\right|^{-1} d v(z) \\
& \quad \leq C \cdot\left(1-\left|w_{i}\right|^{2}\right)^{-\varepsilon}\left(1-\left|w_{j}\right|^{2}\right)^{-\varepsilon} \\
& \int_{D}\left|1-z_{i} \cdot \bar{w}_{i}\right|^{-1} \cdot|z-w|^{-3}\left(1-\left|w_{i}\right|^{2}\right)^{-\varepsilon} d v(w) \leq C \cdot\left(1-\left|z_{i}\right|^{2}\right)^{-\varepsilon}, \\
& \int_{D}\left|1-z_{i} \cdot \bar{w}_{i}\right|^{-1} \cdot|z-w|^{-3}\left(1-\left|z_{i}\right|^{2}\right)^{-\varepsilon} d v(z) \leq C \cdot\left(1-\left|w_{i}\right|^{2}\right)^{-\varepsilon}
\end{aligned}
$$

where $0<\varepsilon<1$.

Note that $H^{\infty}(D)$ is dense in $H^{2}(D)$. By the estimates above, an application of Schur's test [4] yields that $T_{f}(g)=T(g \cdot \bar{\partial} f)$ is bounded from $H^{2}(D)$ to $L^{2}(D)$ and that $\left\|T_{f}\right\|_{2} \leq C \cdot\left\{\left\|\Psi_{1}\right\|_{\infty}+\left\|\Psi_{2}\right\|_{\infty}\right\}$. Q.E.D.

Theorem 2.4. If $f$ satisfies the conditions in Lemma 2.3, then the Hankel operator $H_{f}$ is bounded and $\left\|H_{f}\right\|_{2} \leq C \cdot\left\{\left\|\Psi_{1}\right\|_{\infty}+\left\|\Psi_{2}\right\|_{\infty}\right\}$, where $\Psi_{i}(z)=$ $\left(1-\left|z_{i}\right|^{2}\right) \cdot\left(\partial f(z) / \partial \bar{z}_{i}\right), i=1,2$.

Proof. Note that, for $g \in H^{\infty}(D), f \cdot g \in L^{2}(D)$ is a solution to the equation $\bar{\partial} u=g \cdot \bar{\partial} f$. By Lemmas 2.2 and 2.3, $T_{f}(g)=T(g \cdot \bar{\partial} f) \in L^{2}(D)$ is a solution to the same equation. It is obvious that $f \cdot g-T_{f}(g) \in H^{2}(D)$. Thus, $H_{f}(g)=(I-P)(f \cdot g)=(I-P) T_{f}(g)$. The results follow from Lemma 2.3. Q.E.D.

Theorem 2.5. For $f \in C^{1}(D) \cap L^{2}(D)$, if $\Psi_{i}(z) \rightarrow 0$ as $z \rightarrow \partial D$, then $H_{f}$ is compact.

Proof. Let $D_{m}=\left\{\left(z_{1}, z_{2}\right) \in D:\left|z_{1}\right| \leq 1-1 / m,\left|z_{2}\right| \leq 1-1 / m\right\}$ and $\chi_{m}$ be the characteristic functions of $D_{m}, m=1,2,3, \cdots$. Denote the integral kernel in $(2.5)$ by $L(z, w)$. It is easy to check that

$$
\left|\chi_{m}(w) \cdot L(z, w)\right| \leq C(m)\left(|z-w|^{-3}+\left|z_{1}-w_{1}\right|^{-1}+\left|z_{2}-w_{2}\right|^{-1}\right) .
$$

By the theorem in $\left[9\right.$, p. 360], it follows that the integral operators $Q_{m}$ with kernels $\chi_{m} \cdot L(z, w)$ are compact on $L^{2}(D)$. Note that $\Psi_{i} \rightarrow 0$ as $z \rightarrow \partial D$ for $i=1,2$. Then, for any $\delta>0$, there is an $N$ such that, when $m>N$, we have $\left(1-\chi_{m}\right) \cdot\left(\left|\Psi_{1}\right|+\left|\Psi_{2}\right|\right) \leq \delta$. Note that

$$
T_{f}(g)-Q_{m}(g)=\int_{D} g(w) \cdot\left(1-\chi_{m}(w)\right) \cdot L(z, w) .
$$

By the estimates given in the proof of Lemma 2.3, it follows that $\left\|T_{f}-Q_{m}\right\| \leq$ $C \cdot \delta$. Thus, $Q_{m} \rightarrow T_{f}$ as $m \rightarrow \infty$. The compactness of $Q_{m}$ implies the compactness of $T_{f}$. By the same arguments as in the proof of Theorem 2.4, it follows that $H_{f}$ is compact. Q.E.D.

Note that the Bergman projection is bounded form $L^{p}(D)$ to $H^{p}(D)$ consisting of holomorphic $L^{p}$-functions in $D, 1<p<\infty$. By using the $L^{p}$-version of Schur's test [8, Lemma 5], we have 
Theorem 2.6. If $f$ satisfies the conditions in Theorem 2.4, then $H_{f}$ is bounded from $H^{p}(D)$ to $L^{p}(D)$. If $f$ satisfies the conditions in Theorem 2.5, then $H_{f}$ is compact from $H^{p}(D)$ to $L^{p}(D)$.

\section{MAIN THEOREMS}

Lemma 3.1 [2]. For each $r>0$, there is a sequence of points $\left\{z_{k}\right\} \subset D$ and a constant $M(r)$ such that the Bergman metric balls $\left\{B\left(z_{k}, r / 3\right)\right\}$ form an open cover of $D$, and, for each $z \in D$, there are at most $M(r)$ of $B\left(z_{k}, 3 r\right)$ containing $z$.

Lemma 3.2. Let $r, M(r)$, and $\left\{z_{k}\right\}$ be the same as in Lemma 3.1. There is a partition of unity $\left\{\varphi_{k}\right\}$ subordinate to $B\left(z_{k}, r / 2\right)$ such that $\varphi_{k} \in C^{\infty}(\bar{D})$, $\left|\left(1-\left|z_{i}\right|^{2}\right) \cdot\left(\partial \varphi_{k}(z) / \partial \bar{z}_{i}\right)\right| \leq C$, where $C$ is a constant independent of $z=$ $\left(z_{1}, z_{2}\right) \in D, k \geq 1$, and $i=1,2$.

Proof. Let $\psi \in C^{\infty}\left(\mathbb{C}^{2}\right)$ be a real-valued function with compact support in $B(0, r / 2)$ such that $\psi \geq 0$, and $\psi(z)=1$ on $B(0, r / 3)$. Let $\psi_{k}(z)=$ $\psi\left(\varphi_{z_{k}}(z)\right)$, where $\varphi_{z_{k}}$ is the holomorphic automorphism of $D$ such that $\varphi_{z_{k}}\left(z_{k}\right)$ $=0$. By the invariance of the Bergman metric under biholomorphic mappings and the explicit formula [5] of $\varphi_{z_{k}}$, a direct computation yields that

$$
\left|\left(1-\left|z_{i}\right|^{2}\right) \cdot\left(\partial \psi_{k} / \partial \bar{z}_{i}\right)\right| \leq C .
$$

Write $\varphi_{k}(z)=\psi_{k}(z) /\left(\sum \psi_{k}(z)\right)$. By using Lemma 3.1, one can easily check that $\left\{\varphi_{k}\right\}$ is the desired sequence. Q.E.D.

Now we prove our main theorems.

Proof of Theorem A. (1) $\Rightarrow(2)$. Write $k_{\lambda}(z)=K(z, \lambda) / K(\lambda, \lambda)^{1 / 2}$. From the explicit expression [5] of $K(z, w)$ one can easily check that $k_{\lambda}, k_{\lambda}^{-1} \in H^{\infty}(D)$ and $\left\|k_{\lambda}\right\|_{2}=1$. Note that [2] for $z \in B(\lambda, r),\left|k_{\lambda}(z)\right|^{2} \geq(C \cdot|B(\lambda, r)|)^{-1}$. Thus,

$$
\begin{aligned}
\mid B(\lambda, & r)\left.\right|^{-1} \cdot \int_{B(\lambda, r)}\left|f-k_{\lambda}^{-1} \cdot P\left(f \cdot k_{\lambda}\right)\right|^{2} d v(w) \\
& \leq C \cdot \int_{D}\left|f-k_{\lambda}^{-1} \cdot P\left(f \cdot k_{\lambda}\right)\right|^{2} \cdot\left|k_{\lambda}\right|^{2} d v(w) \\
& =C \cdot\left\|f k_{\lambda}-P\left(f k_{\lambda}\right)\right\|_{2}^{2}=C \cdot\left\|H_{f}\left(k_{\lambda}\right)\right\|_{2}^{2} .
\end{aligned}
$$

It is obvious that $k_{\lambda}^{-1} \cdot P\left(f \cdot k_{\lambda}\right) \in H^{2}(D)$. By the definition of $F_{r}(z)$, it follows that $F_{r}$ is bounded on $D$.

$(2) \Rightarrow(3)$ is trivial. Assume (3). Let $\left\{z_{k}\right\}$ and $\left\{\varphi_{k}\right\}$ be the same as those in Lemma 3.2. By the definition of $F_{r}(z)$, for each $k \geq 1$, there is a function $h_{k} \in H^{2}(D)$ such that

$$
\left|B\left(z_{k}, r\right)\right|^{-1} \cdot \int_{B\left(z_{k}, r\right)}\left|f-h_{k}\right|^{2} d v \leq 4 \cdot F_{r}\left(z_{k}\right)^{2} .
$$

We define $f_{2}=\sum h_{k} \cdot \varphi_{k}, f_{1}=f-f_{2}$. Now we use Luecking's arguments [7] to prove that $f_{1}$ and $f_{2}$ satisfy the conditions in (4). 
If $z \in B\left(z_{i}, r / 2\right) \cap B\left(z_{j}, r / 2\right)$, by Lemma 7 in [2], we have

$$
\begin{aligned}
\mid h_{i}(z) & -\left.h_{j}(z)|\cdot| B(z, r / 2)\right|^{1 / 2} \\
& \leq C \cdot\left(\int_{B(z, r / 2)}\left|h_{i}-h_{j}\right|^{2} d v\right)^{1 / 2} \\
& \leq C \cdot\left(\int_{B\left(z_{i}, r\right)}\left|h_{i}-f\right|^{2} d v\right)^{1 / 2}+C \cdot\left(\int_{B\left(z_{j}, r\right)}\left|h_{j}-f\right|^{2} d v\right)^{1 / 2} \\
& \leq C \cdot\left(F_{r}\left(z_{i}\right)\left|B\left(z_{i}, r\right)\right|^{1 / 2}+F_{r}\left(z_{j}\right)\left|B\left(z_{j}, r\right)\right|^{1 / 2}\right) \\
& \leq C \cdot \sup \left\{F_{r}(w): w \in B(z, 2 r)\right\} \cdot|B(z, 3 r)| .
\end{aligned}
$$

Note that [2] $|B(z, 3 r)| \leq C(r) \cdot|B(z, r / 2)|$. It follows that

$$
\left|h_{i}(z)-h_{j}(z)\right| \leq C \cdot \sup \left\{F_{r}(w): w \in B(z, 2 r)\right\} .
$$

To simplify notation, write $B(z)=B(z, r)$. Then

$$
\begin{aligned}
\left(\int_{B(z)}\left|f_{1}\right|^{2} d v\right)^{1 / 2} & =\left(\int_{B(z)}\left|\sum\left(f-h_{j}\right) \cdot \varphi_{j}\right|^{2} d v\right)^{1 / 2} \\
& \leq \sum\left(\int_{B(z)}\left|\left(f-h_{j}\right) \cdot \varphi_{j}\right|^{2} d v\right)^{1 / 2} \\
& \leq \sum\left(\int_{B\left(z_{j}, r / 2\right) \cap B(z)}\left|f-h_{j}\right|^{2} d v\right)^{1 / 2} \\
& \leq C \cdot M(r) \cdot \sup \left\{F_{r}(w): w \in B(z, 2 r)\right\} \cdot|B(z, 3 r)|^{1 / 2} .
\end{aligned}
$$

The last inequality is because at most $M(r)$ of the $B\left(z_{j}, r / 2\right)$ intersect $B(z)$, and $B\left(z_{j}\right) \subset B(z, 3 r), \beta\left(z, z_{j}\right)<2 r$ when $B\left(z_{j}, r / 2\right) \cap B(z) \neq \varnothing$. Note that [2] $|B(z, 3 r)| \leq C \cdot|B(z, r)|$. Thus,

$$
|B(z)|^{-1} \cdot \int_{B(z, r)}\left|f_{1}\right|^{2} d v \leq C \cdot \sup \left\{F_{r}(w)^{2}: w \in B(z, 2 r)\right\} .
$$

By (3.2) and Lemma 3.1, it is easy to show that $\left\|f_{1}\right\|_{2} \leq C \cdot\left\|F_{r}\right\|_{\infty}$.

Next we estimate $f_{2}$. Fix a point $z \in D$, and let $J$ be the set of integers $j$ such that $z \in B\left(z_{j}, r / 2\right)$. Then $f_{2}(z)=\sum_{j \in J} h_{j}(z) \cdot \varphi_{j}(z)$. For convenience, let us assume that $1 \in J$. Then

$$
f_{2}(z)=h_{1}(z)+\sum_{j \in J}\left(h_{j}(z)-h_{1}(z)\right) \cdot \varphi_{j}(z) .
$$

Note that $h_{1}, h_{j}$ are holomorphic functions. It follows that, for $i=1,2$,

$$
\begin{aligned}
\left|\left(\partial f_{2}(z) / \partial \bar{z}_{i}\right) \cdot\left(1-\left|z_{i}\right|^{2}\right)\right| & =\left|\sum_{j \in J}\left(h_{j}(z)-h_{1}(z)\right) \cdot\left(\partial \varphi_{j}(z) / \partial \bar{z}_{i}\right) \cdot\left(1-\left|z_{i}\right|^{2}\right)\right| \\
& \leq C \cdot M(r) \cdot \sup \left\{F_{r}(w): w \in B(z, 2 r)\right\} .
\end{aligned}
$$

The last inequality holds because there are at most $M(r)$ integers contained in $J$ and $\left|h_{j}-h_{1}\right| \leq C \cdot \sup \left\{F_{r}(w): w \in B(z, 2 r)\right\}$ from $(* *)$, while $\mid\left(\partial \varphi_{j} / \partial \bar{z}_{i}\right) \cdot$ $\left(1-\left|z_{i}\right|^{2}\right) \mid \leq C$. 
From (3.2) and (3.3) it follows that the boundedness of $F_{r}$ implies (4).

Assume (4). By Theorem 2.4, $H_{f_{2}}$ is bounded. By Theorem 8 in [2], $M_{f_{1}}$ is bounded from $H^{2}(D)$ to $L^{2}(D)$. Thus, $H_{f}$ is bounded. Q.E.D.

Proof of Theorem B. (1) $\Rightarrow(2)$. Note that $k_{\lambda} \rightarrow 0$ weakly in $H^{2}(D)$ as $\lambda \rightarrow \partial D$. By (3.1), it follows that $F_{r}(\lambda) \rightarrow 0$ as $\lambda \rightarrow \partial D$.

$(2) \Rightarrow(3)$ is trivial. Assume (3). Note that [2] $\sup _{w \in B(z, 2 r)}\{d(w, \partial D)\} \rightarrow 0$ as $z \rightarrow \partial D$. From (3.2) and (3.3) one can easily get (4). Assume (4). By Theorem 2.5, it follows that $H_{f_{2}}$ is compact. By Theorem 9 in [2], $M_{f_{1}}$ is compact from $H^{2}(D)$ to $L^{2}(D)$. Thus, $H_{f_{1}}$ is compact. This finishes the proof. Q.E.D.

For $1<p<\infty$, write $F_{r, p}(z)=\inf \left\{|B(z, r)|^{-1} \cdot \int_{B(z, r)}|f-h|^{p} d v: h \in\right.$ $\left.H^{p}(D)\right\}$.

Theorem C. Let $f \in L^{p}(D)$. Then the following are equivalent.

(1) $H_{f}$ is bounded from $H^{p}(D)$ to $L^{p}(D)$.

(2) For each $r>0, F_{r, p}$ is bounded on $D$.

(3) For some $r>0, F_{r, p}$ is bounded on $D$.

(4) $f$ admits a decomposition $f=f_{1}+f_{2}$ with $f_{2} \in C^{1}(D) \cap L^{p}$ and $f_{1} \in L^{p}(D)$ such that, for each $1 \leq i \leq n,\left(\partial f_{2} / \partial \bar{z}_{i}\right) \cdot\left(1-\left|z_{i}\right|^{2}\right) \in L^{\infty}(D)$ and, for some $r>0$,

$$
G_{r, p}(z)=|B(z, r)|^{-1} \cdot \int_{B(z, r)}\left|f_{1}\right|^{p} d v \in L^{\infty}(D) .
$$

Theorem D. Let $f \in L^{p}(D)$. Then the following are equivalent.

(1) $H_{f}$ is compact from $H^{p}(D)$ to $L^{p}(D)$.

(2) For each $r>0, F_{r, p}(z) \rightarrow 0$ as $z \rightarrow \partial D$.

(3) For some $r>0, F_{r, p}(z) \rightarrow 0$ as $z \rightarrow \partial D$.

(4) $f$ admits a decomposition $f=f_{1}+f_{2}$ with $f_{2} \in C^{1}(D) \cap L^{p}$ and $f_{1} \in L^{p}(D)$ such that, for each $1 \leq i \leq n,\left(\partial f_{2} / \partial \bar{z}_{i}\right) \cdot\left(1-\left|z_{i}\right|^{2}\right) \rightarrow 0$ as $z \rightarrow \partial D$ and, for some $r>0, G_{r, p}(z) \rightarrow 0$ as $z \rightarrow \partial D$.

Since the proofs of Theorems C and D are the same as those of Theorems A and B except for obvious modifications, we omit the details here.

Finally, we note that our results and methods can be extended to the Hankel operators on the weighted Bergman spaces with weight functions $\left(1-\left|z_{1}\right|^{2}\right)^{\alpha}$. $\left(1-\left|z_{2}\right|^{2}\right)^{\beta}, \alpha>-1, \beta>-1$, on the unit polydisc.

\section{ACKNOWLEDGMENT}

I thank Professor L. A. Coburn for his good advice. I am grateful to the referee for his good suggestions.

\section{REFERENCES}

1. S. Axler, The Bergman space, the Bloch space and commutators of multiplication operators, Duke Math. J. 53 (1986), 315-332.

2. D. Bekolle, C. A. Berger, L. A. Coburn, and K. Zhu, BMO in the Bergman metric on bounded symmetric domains, J. Funct. Anal. 93 (1990), 310-350.

3. P. Charpentier, Formules explicites pour les solutions minimales de l'equation $\bar{\partial} u=f$ dans la boule et dans le polydisque de $\mathbb{C}^{n}$, Ann. Inst. Fourier (Grenoble) 30 (1980), 121-153. 
4. P. Halmos and V. Sunder, Bounded integral operators on $L^{2}$ space, Springer-Verlag, New York and Berlin, 1978.

5. S. G. Krantz, Function theory of several complex variables, Wiley, New York, 1982.

6. $\mathrm{H}$. Li, Hankel operators on the Bergman spaces of strongly pseudoconvex domains, preprint, 1991.

7. D. Luecking, Characterizations of certain classes of Hankel operators on the Bergman spaces of the unit disk, preprint, 1991.

8. D. Phong and E. M. Stein, Estimates for the Bergman and Szego projections on strongly pseudoconvex domains, Duke Math. J. 44 (1977), 695-704.

9. R. M. Range, Holomorphic functions and integral representations in several complex variables, Springer-Verlag, New York, 1986.

10. H. Skoda, Valeurs au bord pour les solutions de l'operator d" et caracterisation des zeros des fonctions de la classe de Nevanlinna, Bull. Soc. Math. France 104 (1976), 225-299.

11. K. Stroethoff, Compact Hankel operators on the Bergman space, Illinois J. Math. 34 (1990), 159-174.

Department of Mathematics, State University of New York at Buffalo, Buffalo, New YoRK 14214

E-mail address: hli@cs.buffalo.edu 